PAPER

\title{
The probability of middle cerebral artery MRA flow signal abnormality with quantified CT ischaemic change: targets for future therapeutic studies
}

\author{
P A Barber, A M Demchuk, M D Hill, J H Warwick Pexman, M E Hudon, R Frayne, A M Buchan
}

J Neurol Neurosurg Psychiatry 2004;75:1426-1430. doi: 10.1136/jnnp.2003.029389

See end of article for authors' affiliations

Correspondence to:

P A Barber, Assistant

Professor, Department of

Clinical Neurosciences,

University of Calgary,

Institute for Biodiagnostics

(West), Room 153, 3330

Hospital Drive, Calgary,

Canada AB T2N $4 \mathrm{N1}$;

pabarber@ucalgary.ca

Received

30 September 2003

In revised form

21 December 2003

Accepted

23 December 2003

\begin{abstract}
Objectives: In this study we define the probability of vascular abnormality in the middle cerebral artery (MCA) territory according to the extent of ischaemic change seen using computed tomography (CT). We assessed the sensitivity and specificity of the hyperdense middle cerebral artery (HMCA) and the "dof" sign using magnetic resonance angiography (MRA).

Methods: Patients presenting with ischaemic stroke had a CT scan $(<6 \mathrm{~h})$ prior to MRI $(<7 \mathrm{~h})$. A quantitative CT scoring system (ASPECTS) was applied to CT and diffusion weighted images (DWI) at baseline and follow up (24 h) by five independent observers. The presence of HMCA and the MCA "dot" sign was also evaluated. An expert reader assessed the 3D time of flight (TOF) MRA in the anterior circulation for areas of decreased vascular signal in the MCA territory, with an absent signal taken to represent severely reduced or absent flow.

Results: A total of 100 consecutive patients had baseline CT and MR scans. The median NIHSS was 9. The median CT ASPECTS was 8 and equalled the median DWI ASPECTS. There were a total of $10 \mathrm{HMCA}$ and 19 MCA "dot" signs, with four patients having both HMCA and "dot" signs. A total of 47 MRA flow signal abnormalities were observed in the anterior circulation.

Conclusions: In the absence of accessible neurovascular imaging, the extent of CT ischaemia (ASPECTS) is a strong predictor of vascular occlusion. The CT hyperdense artery signs have a high positive predictive value but low negative predictive value.
\end{abstract}

schaemic stroke is currently the third leading cause of death in the Western world and the leading cause of acquired adult disability. ${ }^{1}$ In Caucasians, occlusion of extra- or intracranial cerebral arteries by thromboembolic material can be demonstrated early after stroke onset in $80 \%$ of stroke patients ${ }^{2}$ making thrombolysis a rational therapeutic choice. Intravenous tissue plasminogen activator (tPA) remains the only drug and route of administration approved in North America and Europe for acute stroke treatment based on the results of the NINDS tPA trials and is recommended in all disabling ischaemic stroke patients fulfilling the clinical criteria of the NINDS tPA study. ${ }^{3}$ Other thrombolytic trials have produced neutral results. ${ }^{4-6}$ These trials had a common methodological design, and enrolled patients with ischaemic stroke by excluding intracranial haemorrhage using computed tomography but did not use any form of neurovascular imaging to identify a large artery occlusion (either extra- or intracranial). In contrast, the ProLyse for Acute Cerebral Thromboembolism trial (PROACT-II $^{7}$ randomised a more homogenous population of acute stroke patients with angiographically proven middle cerebral artery occlusions and demonstrated a positive result.

The reality is that a simpler approach relying on clinical parameters and non invasive technology may facilitate more appropriate and widespread use of thrombolysis. The three objectives of this study were: (1) to describe the frequency of magnetic resonance angiographic (MRA) flow signal abnormalities of the internal carotid artery and/or middle cerebral artery in an acute ischaemic stroke population with disabling deficits; (2) to define the probability of intracranial abnormality in the anterior circulation by a quantitative topographical scoring system, the Alberta Stroke Program Early CT Score (ASPECTS) ${ }^{8}$; and (3) to determine the sensitivity and specificity of the hyperdense middle cerebral artery (HMCA)and "dot" signs using MRA as the gold standard. ${ }^{10}{ }^{11}$

We speculated that MRA would provide similar anatomical localisation to formal cerebral angiography, that the HMCA sign represented middle cerebral artery occlusion in the main stem of the artery, and the MCA "dot" sign would correlate with occlusion either in the distal portion of the middle cerebral artery or in the M2 branches. ${ }^{12}{ }^{13}$

\section{METHODS}

The period of patient recruitment was from October 1999 to July 2001. The principal inclusion criterion for the study was acute disabling ischaemic stroke, measurable by the National Institute of Health Stroke Scale (NIHSS) score, within $6 \mathrm{~h}$ of onset. All patients sequentially had a CT brain scan within $6 \mathrm{~h}$ followed by MRI within $7 \mathrm{~h}$ of onset. Some patients who met accepted criteria were treated with intravenous thrombolysis and the ensuing MR study was performed while the tPA infusion was running. Information collected on subjects included: demographic details; an NIHSS score on initial presentation; time of symptom onset and time at each imaging procedure; and functional outcome at 3 months assessed by the modified Rankin scale (mRS). The mRS was evaluated by a nurse practitioner not involved with the initial individual patient care. Favourable outcome was defined as

Abbreviations: ACAs, anterior cerebral artery; ASPECTS, Alberta Stroke Program Early CT Score; BA, basilar artery; CT, computed tomography; DWI, diffusion weighted imaging; FLAIR, fluid attenuated inversion recovery; HMCA, hyperdense MCA; ICA, internal carotid artery; MCA, middle cerebral artery; MRA, magnetic resonance angiography; mRS, modified Rankin scale; NIHSS, National Institute of Health Stroke Scale; PCA, posterior cerebral artery; PROACT-II, Prolyse for Acute Cerebral Thromboembolism trial; IPA, tissue plasminogen activator 
independence (mRS of $0-2$ ). The study was approved by the local ethics committee, and was part of a study to compare CT with diffusion weighted imaging (DWI) in acute ischaemic stroke. $^{14}$

All CT scans were performed on fourth generation scanners and were considered to be optimal quality. The standard CT scan protocol was $5 \mathrm{~mm}$ slice thickness without contrast enhancement, $120 \mathrm{kV}, 170 \mathrm{~mA}$ at $2 \mathrm{~s}$, contrast favoured algorithm, inferior orbitomeatal baseline, filmed at appropriate window width and level setting of 80/40 Hounsfield units. $^{9}$

MR imaging was performed on a $3 \mathrm{~T}$ scanner (General Electric Medical Systems, Waukesha, WI) equipped with a standard head quadrature imaging coil and high-speed gradients. The rationale for using $3 \mathrm{~T}$ MRI rather than $1.5 \mathrm{~T}$ MRI is that the former has better signal to noise ratio. ${ }^{15}$ The imaging sequences included sagittal Tl-weighted, axial diffusion-weighted, and perfusion-weighted imaging, fluid attenuated inversion recovery (FLAIR) imaging, and axial multi-slab 3D time of flight MR angiography (MRA). The isotropic diffusion-weighted images were obtained using a single shot EPI ( $b=1000 \mathrm{~s} / \mathrm{mm}^{2}, 19-5 \mathrm{~mm}$ thick slices $)$. The imaging parameters for the conventional two-slab 3D TOF sequence were $24 / 3.3 / 15^{\circ}$ (TR/TE/flip angle), acquisition band with of $12.5 \mathrm{~Hz}$, and a $240 \times 192 \times 46 \mathrm{~mm}$ acquired volume with a $256 \times 192 \times 42$ matrix, which was then reconstructed to a $512 \times 512 \times 84$ matrix. Axial slabs were prescribed from approximately the level of the skull base to the circle of Willis.

Each axial imaging technique (that is, CT and MRI) was read independently by each of five readers, blinded to all clinical and patient information apart from side of stroke symptoms. The rationale for this was to make the imaging interpretation more clinically relevant. The images were read in the following order: baseline CT, FLAIR, and DWI followed by the follow up CT, FLAIR, and DWI. Each imaging modality/technique was interpreted in isolation of other modalities, with a minimum period of 1 week between readings of scans by the same individual.

Each axial CT image (CT) was scored using the Alberta Stroke Program Early CT Score (ASPECTS). ${ }^{89}$ A normal CT scan received an ASPECTS of 10 points; a single point was subtracted for an area of ischaemic change as defined by von Kummer, ${ }^{16}{ }^{17}$ which included any or all parenchymal hypoattenuation, loss of grey white differentiation, and focal brain swelling. Parenchymal hypoattenuation was defined as a region of abnormally decreased attenuation of brain structures relative to attenuation of other parts of the same structures or of the contralateral hemisphere. Focal brain swelling was defined as any focal narrowing of the cerebrospinal fluid space due to compression by adjacent brain structures producing effacement of the cortical sulci or ventricular compression. A score of 0 indicated diffuse ischaemic involvement throughout the MCA territory. The $24 \mathrm{~h}$ CT scan was read under the same conditions.

The presence of an HMCA sign ${ }^{10}$ and a sylvian fissure MCA "dot" sign ${ }^{11}$ was assessed. The HMCA was defined as an MCA denser than its counterpart and any other vascular structure excluding obvious calcification. The MCA "dot" sign was defined as the hyperdensity of an arterial structure (seen as a dot) in the sylvian fissure relative to the contralateral side or to other vessels within the sylvian fissure.

The MR angiogram was assessed by a single expert reader blind to all information apart from stroke symptom side, firstly at baseline, and then at the follow up at $24 \mathrm{~h}$. The reasons for using MRA as the preferred imaging modality were its non invasiveness, the avoidance of radiation contrast agents, and its superior accuracy compared to other non invasive vascular techniques such as transcranial Doppler.
For each non contrast examination the collapsed maximum intensity projection and source images were reviewed. The reader detailed the vascular abnormalities by the vascular signal intensity in the following extra- and intracranial vessels: the left and right internal carotid artery (ICA), the basilar artery (BA), the anterior cerebral arteries (ACAs), the left and right main stem of the middle cerebral artery segments (M1) and the MCA branch division (M2) regions of the MCA, and the left and right main posterior cerebral artery (PCA) segments.

\section{STATISTICAL ANALYSIS}

Data are reported using descriptive statistics. Baseline and follow up CT and DWI ASPECTS values were derived as the median of the results of the five observers. Comparisons of proportions, sensitivity and specificity, relative risks, and confidence intervals were assessed using $2 \times 2$ tables and exact methods. The presence of either M1 or M2 MRA flow signal abnormality was used to perform the sensitivity calculation. Logistic regression was used to adjust for baseline stroke severity in the assessment of the association between ASPECTS and arterial occlusion. Functional outcome was dichotomised into independence (mRS 0-2) and dependence or death (mRS 3-6).

\section{RESULTS}

A total of 100 consecutive patients were enrolled into the study, of whom $69 \%$ were male, and the mean (SD) age was 68 (13.9) years. The median NIHSS was 9 (interquartile range 3-16). A total of 39 patients received tPA: 33 patients received intravenous tPA alone, and six received a combined approach of intravenous tPA followed by intra-arterial thrombolysis into the angiographically defined thrombus. ${ }^{18}$ Of the patients 60\% were independent (modified Rankin score $0-2$ ) at 3 months (two patients were lost to follow up at 3 months). There were nine deaths, of which two were due to tPA-related fatal intracerebral haemorrhages.

The median baseline CT ASPECTS was 8, which was the same as the median baseline DWI ASPECTS. A total of $67 \%$ (95\% confidence interval (CI) 0.59 to 0.78 ) of the patients had CT ischaemic change (ASPECTS $\leqslant 9$ ), while 79\% (95\% CI 0.70 to 0.87$)$ of the DWI scans identified areas of hyperintense signal (DWI ASPECTS $\leqslant 9$ ). Six patients $(6 \%)$ had evidence of posterior circulation ischaemia on baseline DWI with or without coincident anterior circulation stroke. The mean (SD) time from symptom onset to CT was 117 (70) min compared to 219 (80) min to MR imaging (a mean (SD) difference of 102 (51) min, p=0.0002). On CT, there were 10 hyperdense MCA signs and 19 MCA "dot" signs (four patients had both hyperdense MCA and "dot" signs) (fig 1). There were a total of 47 MRA flow signal abnormalities in the anterior circulation, of which 14 involved the ICA, either with M1 (five) or M2 (seven) alone, or both M1 and M2 (two). A total of 18 MRA abnormalities involved M1 (without ICA involvement), and 15 M2 MRA abnormalities (without involvement of either ICA or Ml). The sensitivity and specificity of the hyperdense MCA and MCA "dot" sign are presented in table 1 .

Both ASPECTS value (fig 2) and baseline NIHSS score predicted the presence of an intra- or extracranial occlusion. The relative risk of an occlusion on 3D TOF with a baseline NIHSS $\geqslant 10$ was 2.22 ( $95 \%$ CI 1.39 to 3.56). After adjusting for baseline stroke severity, for each decrement of one ASPECTS point, the odds of an occlusion involving the internal carotid artery, M1 or M2 branches rose 2.7 fold (95\% CI 1.8 to 4.1 ). Alternately, when the baseline CT ASPECTS was less than or equal to 7, the probability of occlusion on subsequent MR angiography was 0.88 (95\% CI 0.72 to 0.97$)$ for a relative risk of occlusion of 3.27 ( $95 \%$ CI 2.2 to 5.0 ). No interaction was 

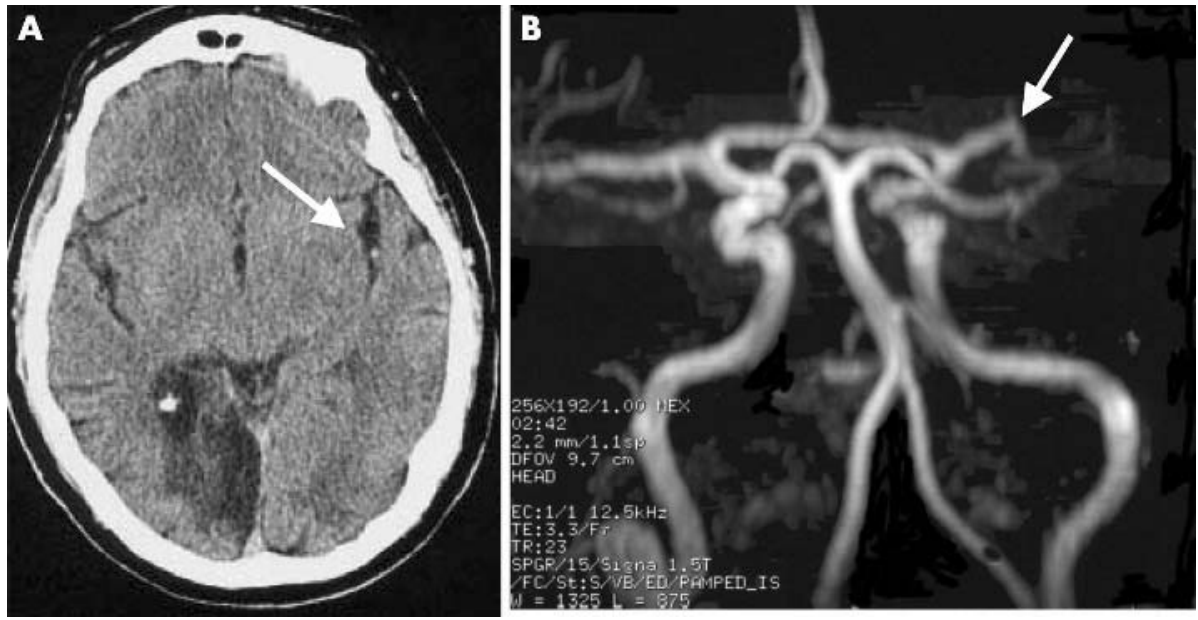

Figure 1 (A) A baseline CT scan performed on a 64 year old male patient who presented with acute dysphasia and right sided weakness, $2 \mathrm{~h}$ into the symptoms. A hyperdense MCA "dot" sign (arrow) suggests a thromboembolic occlusion of the M2 branch artery. There are also early CT ischaemic changes in the posterior insula and posterior temporal lobe. Incidentally there is an old right PCA stroke. (B) MRA confirming a flow signal abnormality suggesting either occlusion or slow flow in the M2 branch. The patient received intravenous $\mathrm{PAA}$, but unfortunately was functionally dependent at 3 months.

detected between ASPECTS value and baseline NIHSS score (likelihood ratio test, $\mathrm{p}=0.70$ ), meaning the ability of ASPECTS to predict the MRA flow signal abnormality is not dependent on the NIHSS score.

\section{DISCUSSION}

Neurovascular imaging can assist in the appropriate selection of stroke patients for thrombolysis. ${ }^{19}$ Patients without occlusions receiving treatment with thrombolysis are possibly exposed to risk without obvious potential benefit. Results from the PROACT-II study suggest that, among patients with confirmed intracranial occlusion, the time window for useful intervention is longer than $3 \mathrm{~h}$ following the onset of symptoms.

In this cohort of consecutive patients stroke severity (median NIHSS 9) was not disimilar to that of patients enrolled in either the ECASS II or ATLANTIS B thrombolysis clinical trials. ${ }^{45}$ Yet only $47 \%$ of cases in this study had demonstrable MRA flow signal abnormalities in the MCA vascular territory. The most striking observation from this study was that ASPECTS predicts MRA flow signal abnormality independently of the NIHSS score. As the ASPECTS value decreased the probability of an MRA flow signal abnormality increased such that all cases with an ASPECTS of 5 and below had an MRA flow abnormality. When the CT scan was normal (ASPECTS 10) the prevalence of MRA flow abnormality in the anterior circulation was small ( $11 \%)$. Both ASPECTS and NIHSS were independent predictors of occlusion, and therefore, may be important clinical indices in predicting vascular occlusion. The relationship between NIHSS and MRA flow signal abnormalities has been previously reported. ${ }^{20}$

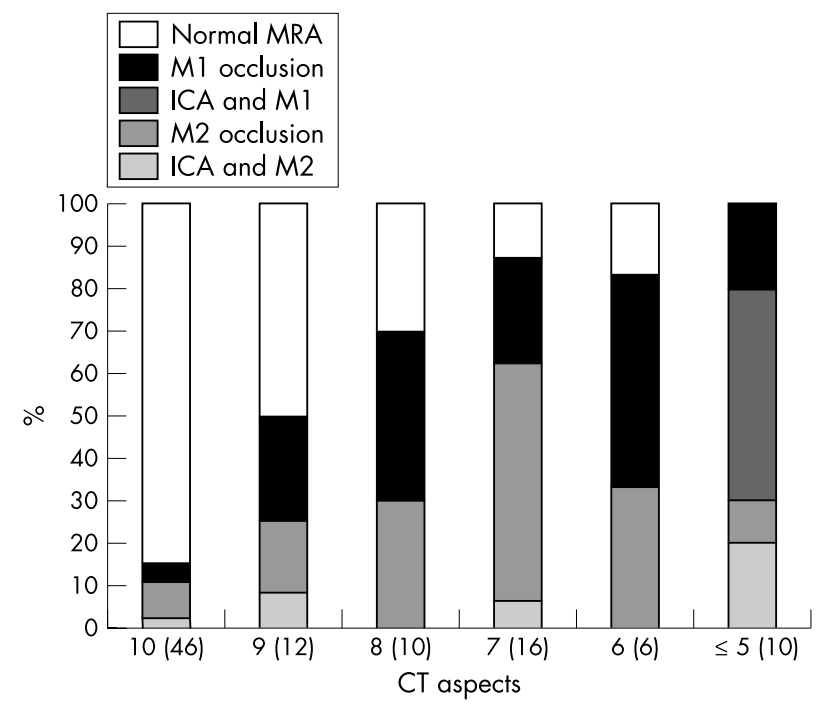

Figure 2 A histogram of baseline CT ASPECTS (horizontal axis) values and the probability of MRA flow signal abnormality (\%, vertical axis) in the anterior circulation. The numbers in parentheses refer to the number in each ASPECTS group. ICA, internal carotid artery; MI branch, middle cerebral artery main stem; $M 2$ branch, main division of $M 1$ branch.

The HMCA sign has been associated with severe neurological deficit, extensive infarction in the MCA territory, and poor outcome ${ }^{21}$ and correlates well with MCA occlusion based on angiography. ${ }^{12}{ }^{13}$ The recently described MCA "dot" sign $^{11}$ correlates with distal MCA occlusion identified by

\begin{tabular}{|c|c|c|c|c|}
\hline & & & Sensitivity & Specificity \\
\hline Ml stem & HMCA sign present & HMCA sign absent & & \\
\hline Occluded, $n(n=25)$ & 7 & 18 & 0.28 & 0.96 \\
\hline Patent, $n(n=75)$ & 3 & 72 & $\begin{array}{l}(95 \% \mathrm{Cl} \\
0.12 \text { to } 0.49)\end{array}$ & $\begin{array}{l}(95 \% \mathrm{Cl} \\
0.88 \text { to } 0.99)\end{array}$ \\
\hline M2 branch & $\begin{array}{l}\text { MCA "dot" sign } \\
\text { present }\end{array}$ & $\begin{array}{l}\text { MCA "dot" sign } \\
\text { absent }\end{array}$ & & \\
\hline Occluded, $n(n=24)$ & 11 & 13 & 0.46 & 0.89 \\
\hline Patent, $n(n=76)$ & 8 & 68 & $\begin{array}{l}(95 \% \mathrm{Cl} \\
0.26 \text { to } 0.67)\end{array}$ & $\begin{array}{l}(95 \% \mathrm{Cl} \\
0.80 \text { to } 0.95)\end{array}$ \\
\hline
\end{tabular}


conventional angiography. We now report the sensitivity and specificity of these signs using 3D TOF MRA as the gold standard. In accordance with previous angiographic studies, ${ }^{22}$ the sensitivity of both these signs is in the poor to moderate range, but both have very high specificity, confirming that when present they have a high positive predictive value for thromboembolic occlusion.

MRA provides anatomical and physiological evidence of flow through the major collateral pathways, and allowed non invasive correlation of the CT vascular signs. Conventional angiography remains the gold standard for assessing the collateral pathways and is consistent with anatomical studies. ${ }^{23}$ But the risk of inducing stroke in this particular patient group may be similar to or higher than the complication rate in the Asymptomatic Carotid Surgery Trial of $1 \%{ }^{24}$ Time of flight angiography allows the arteries of the circle of Willis to be examined by the endogenous contrast of flow-related spin enhancement and a flow related abnormality can indicate either an absence of flow or slow flow. The rationale for the use of MRA as the gold standard was its safety (cf formal cerebral angiography), and avoidance of radiation and contrast bolus as with CTA. In addition its accuracy is probably superior to other techniques such as transcranial Doppler. ${ }^{25}$ In a study comparing TOF MR angiography of carotid stenosis, flow void artefacts represent severe carotid stenosis when compared to formal angiography. ${ }^{26}$ The major drawback of this method is the depiction of the distal intracranial vessels which may account for some of the under-reporting of distal MCA branch abnormalities in this study. ${ }^{27}$

Previous data support the concept that stroke patients can be selected on the basis of stroke severity and quantified CT ischaemia. ${ }^{4-8} 2829$ There is mounting evidence that the extent of CT ischaemia is very important in determining response to therapy. In a post hoc analysis of ECASS $I_{,}{ }^{29}$ patients with signs of ischaemia involving $\leqslant 1 / 3$ MCA benefited from tPA treatment when compared to placebo (OR 3.43, 95\% CI 1.61 to 7.33 ). The benefit was less clear for patients with normal CT scans or parenchymal hypodensity involving >1/3 MCA, and did not exclude the possibility of harm.

These data combined with the current work support the idea of a therapeutic trichotomy in acute stroke patients: (1) the normal CT scan (low prevalence of middle cerebral artery occlusion) may indicate patients who will improve spontaneously; (2) the CT scan with early but not extensive ischaemic changes (ASPECTS 7-9; high prevalence of MCA occlusion) may indicate the ideal thrombolysis candidate; and (3) the CT scan with extensive CT ischaemic change (high prevalence of MCA occlusion) may indicate the patient least likely to benefit from thrombolysis. This stratification may change with time from stroke onset. Therefore, we propose that successful tPA adminstration for acute stroke depends on careful clinical assessment, combined with selection of patients with a CT scan showing moderate rather than extensive ischaemic change. This group of patients have a high probability of large artery occlusion with associated "tissue at risk", and therefore may benefit most from thrombolysis. This concept would require further validation before it impacts patient care but a systematic approach to the assessment of non contrast CT with ASPECTS may provide a useful surrogate in future thrombolysis trials.

New CT techniques such as CT angiography, CT perfusion, and post contrast and blood pool analysis are being increasingly evaluated in clinical practice: the latter has been shown to increase the visualisation of ischaemic areas by $40 \%{ }^{30}$ However, the functional outcome following tPA administration is time dependent, ${ }^{31}$ and additional contrast enhanced CT techniques, as with MRI, may delay initiation of treatment with no additional benefit.

\section{ACKNOWLEDGEMENTS}

The authors thank Kathryn Werdal and Andrea Cole-Haskaynes for administrative aid in organising films for review.

\section{Authors' affiliations}

P A Barber, A M Demchuk, M D Hill, J H Warwick Pexman, M E Hudon, R Frayne, A M Buchan, Calgary Stroke Program, Department of Clinical Neuroscience, Seaman Family Magnetic Research Centre, 1403-29 St. NW, Calgary, AB, Canada

M D Hill, Department of Medicine and Department of Community Health Sciences, University of Calgary, Calgary, AB, Canada

M E Hudon, Department of Clinical Radiology, University of Calgary, Calgary, AB, Canada

R Frayne, Department of Radiology, University of Calgary, Calgary, $A B$, Canada

This study was supported by the Alberta Foundation for Health Research. Dr Barber was supported by the Canadian Institute of Health Research, the Heart and Stroke Foundation of Canada, and the Alberta Heritage Foundation for Medical Research. Dr Hill was supported by the Heart and Stroke Foundation of Alberta, NWT, Nunavut and the Canadian Institutes for Health Research. Dr Demchuk was supported by the Alberta Heritage Foundation for Medical Research and the Canadian Institute of Health Research. Dr Frayne was supported by Heart and Stroke Foundation of Canada and the Alberta Heritage Foundation for Medical Research. Dr Buchan was supported by the Heart and Stroke Foundation of Canada, and the Alberta Heritage Foundation for Medical Research.

Competing interests: none declared

\section{REFERENCES}

1 The National Institute of Neurological Disorders and Stroke rt-PA Stroke Study Group. Tissue plasminogen activator for acute ischemic stroke. N Engl J Med 1995;333:1581-7.

2 del Zoppo GJ, Poeck K, Pessin MS, et al. Recombinant tissue plasminogen activator in acute thrombotic and embolic stroke. Ann Neurol 1992;32:78-86.

3 The NINDS t-PA Stroke Study Group. Generalized efficacy of t-PA for acute stroke. Subgroup analysis of the NINDS t-PA stroke trial. Stroke 1997;28:2119.

4 Hacke W, Kaste M, Fieschi C, et al, for the ECASS Study Group. Intravenous thrombolysis with recombinant tissue plasminogen activator for acute hemispheric stroke: The European Cooperative Acute Stroke Study (ECASS) JAMA 1995;274:1017-25.

5 Hacke W, Kaste M, Fieschi C, et al. Randomised double blind placebocontrolled trial of thrombolytic therapy with intravenous alteplase in acute ischaemic stroke (ECASS II). Lancet 1998;352:1245-51.

6 Clark WM, Wissman S, Albers GW, et al. Recombinant tissue-type plasminogen activator for ischemic stroke $3-5$ hours after symptom onset. The ATLANTIS Study: a randomised controlled trial. JAMA 1999;282:2019-26.

7 Furlan A, Higashida R, Wechsler L, et al, for the PROACT investigators. Intra-arterial prourokinase for acute ischemic stroke. The PROACT II Study: a randomised controlled trial. JAMA 1999;282:2003-11.

8 Barber PA, Demchuk AM, Zhang J, et al. Hyperacute stroke: the validity and reliability of a novel quantitative $\mathrm{CT}$ score in predicting outcome prior to thrombolytic therapy. Lancet 2000;355:1670-4.

9 Pexman JHW, Barber PA, Hill MD, et al. Use of the Alberta Stroke Program Early CT Score (ASPECTS) for assessing CT scans in patients with acute stroke. AJNR Am J Neuroradiol 2001;22:1534-42.

10 Leys D, Pruvo JP, Godefroy O, et al. Prevalence and significance of hyperdense midd́le cerebral artery in acute stroke. Stroke 1992;23:317-24

11 Barber PA, Demchuk AM, Zhang J, et al. The hyperdense sylvian fissure MCA "dot" sign". A CT marker of acute cerebral ischemia. Stroke 2001;32:84-8.

12 Bastianello S, Pierallini A, Colonnese C, et al. Hyperdense middle cerebral artery CT sign. Comparison with angiography in the acute phase of ischemic supratentorial infarction. Neuroradiology 1991;33:207.

13 Leary MC, Saver JL, Kidwell CS, et al. Validation of the CT MCA dot sign: an angiographic correlation study (abstract). Stroke 2002;33:342.

14 Barber PA, Demchuk AM, Hill MD, et al. A comparison of CT versus MR imaging in acute stroke using ASPECTS: will the "new" replace the "old" as the preferred imaging modality? (abstract). Stroke 2001;32:325.

15 Frayne R, Goodyear BG, Dickhoff P, et al. Magnetic resonance imaging at 3.0 Tesla: challenges and advantages in clinical neurological imaging. Invest Radiol 2003;38(7):385-402

16 von Kummer R, Allen KL, Holle R, et al. Acute stroke: usefulness of early CT findings before thrombolytic therapy. Radiology 1997;205:327-33.

17 von Kummer R. Acute stroke: how to improve CT detection and avoid errors in radiology. Radiology 2000;216(3):920-23.

18 Hill MD, Barber PA, Demchuk AM, et al. Acute IV-IA revascularization therapy for severe ischemic stroke. Stroke 2002;33:279-82.

19 Caplan LR, Mohr JP, Kistler JP, et al. Clinical debate: should thrombolytic therapy be the first-line treatment for acute ischemic stroke? N Engl Med 1997;337:1309-13. 
20 Derex L, Nighoghossian N, Hermier $M$, et al. Early detection of cerebral arterial occlusion on magnetic resonance angiography: predictive value of the baseline NIHSS score and impact on neurological outcome. Cerebrovasc Dis 2002;13:225-9.

21 Manelfe $C$, Larrue $V$, von Kummer R, et al. Association of hyperdense middle cerebral artery sign with clinical outcome in patients with tissue plasminogen activator. Stroke 1999;30:769-72.

22 Wolpert SM, Bruckmann H, Greenlee R, et al. Neuroradiologic evaluation of patients with acute stroke treated with recombinant tissue plasminogen activator. The rt-PA Acute Stroke Study Group. AJNR Am J Neuroradiology 1993;14:3-13

23 Sacco RL, Ellenberg JH, Mohr JP, et al. Infarcts of undetermined cause: the NINCDS Stroke Data Bank. Ann Neurol 1989;25(4):382-90.

24 Executive Committee for the Asymptomatic Carotid Atherosclerosis Study. Endarterectomy for asymptomatic carotid artery stenosis. JAMA 1995;273(18): 1421-8.

25 Suwanwela NC, Phanthumchinda K, Suwanwela N. Transcranial Doppler sonography and CT angiography in patients with atherothrombotic middle cerebral artery stroke. AJNR Am J Neuroradiol 2002;23(8):1352-5.
26 Nederkoorn PJ, Elgersma OE, van der Graaf Y, et al. Carotid artery stenosis: accuracy of contrast-enhanced MR angiography for diagnosis. Radiology 2003;228(3):677-82.

27 Yang JJ, Hill MD, Morrish WF, et al. Comparison of pre- and postcontrast 3D time-of-flight $M R$ angiography for the evaluation of distal intracranial branch occlusions in acute ischemic stroke. ANNR Am J Neuroradiol 2002;23(4):557-67.

28 Hill MD, Rowley HA, Adler F, et al for the PROACT-II Investigators. Selection of acute ischaemic stroke patients for intra-arterial thrombolysis with prourokinase using ASPECTS. Stroke 2003:34:1925-31.

29 Von Kummer R, Weber J. Brain and vascular imaging in acute ischemic stroke: the potential of computed tomography. Neurology 1997;49(suppl 4):S52-5.

30 Ezzeddine MA, Lev MH, McDonald CT, et al. CT angiography with whole brain perfused blood volume imaging: added clinical value in the assessment of acute stroke. Stroke 2002;33:959-66.

31 Marler JR, Tilley BC, Lu M, et al. Early stroke treatment associated with better outcome: the NINDS rt-PA Stroke Study. Neurology 2000;55:1649-55 


\section{PostScript}

\section{LETTERS}

\section{Possible transcallosal seizure induction by paired pulse transcranial magnetic stimulation in a patient with frontal lobe epilepsy} cranial magnetic stimulation (TMS) has been reported in normal subjects and by single pulse TMS close to the epileptic focus in patients with epilepsy.

\section{Case report}

We report an 18 year old patient with right frontal lobe epilepsy due to paramedian focal cortical dysplasia (FCD). The patient's usual seizure semiology consisted of a somatosensory aura of the left hand followed by a tonic seizure of the left arm which evolved to a bilateral asymmetrical tonic seizure without loss of consciousness. In the two years preceding the study (see below) he had rare night-time seizures only. His antiepileptic medication consisted of levetiracetam $500 \mathrm{mg}$, phenobarbital $25 \mathrm{mg}$, and carbamazepine $1600 \mathrm{mg}$ daily.

During presurgical videoelectroencephalogram (video-EEG) monitoring, interictal EEG showed right frontotemporal spikes. Ictal EEG revealed seizure patterns with a right frontal onset. Magnetic resonance imaging (MRI) showed FCD in the right superior frontal gyrus extending into the right precentral gyrus (fig 1A). Neurological examination was normal.

\section{Transcranial magnetic stimulation} a protocol described previously ${ }^{2}$ to evaluate
Seizure induction by high frequency trans-

The patient participated in a TMS study using

intracortical excitability of both motor cortices (Ml). The study was approved by the local ethics committee, and the patient gave written informed consent.

We used a focal $70 \mathrm{~mm}$ figure of eight coil connected to two magnetic stimulators via a BiStim module (Magstim Company, Dyfed, UK). Surface electromyography (EMG) was recorded from the contralateral abductor digiti minimi muscle (ADM) of the hand.

TMS commenced over the left Ml contralateral to the epileptic focus with the coil placed over the Ml hand area. First, motor thresholds (RMT, AMT) and cortical induced silent period at an intensity of $110 \%$ RMT were evaluated. Next, paired pulse TMS (conditioning stimulus set at $38 \%$ of maximum stimulator output, second stimulus $60 \%$ of stimulator output) was started on the left Ml with a train of paired pulses with ISI $2,3,10$, and $15 \mathrm{~ms}$ in a random order.

After 65 stimuli, the patient noticed that his habitual somatosensory aura of the left hand followed by myoclonic jerks of the left forearm (mainly biceps brachii muscle and forearm flexor muscles) was triggered by each stimulus, contralateral to the epileptogenic zone but ipsilateral to the cortical stimulation. The jerks were triggered by both single and paired stimuli at all ISI and rapidly involved both arms. These motor phenomena were different from the typical seizure semiology. EMG recordings of the ADM showed movement artefacts 63-75 ms after the MEP (fig 1B). The TMS was immediately interrupted, which aborted the myoclonus at once.

The TMS data of the left hemisphere showed increased motor thresholds, prolonged cortical induced silent period, markedly decreased intracortical inhibition, and increased facilitation compared with 20 controls $^{2}$ (percentiles of the patient's measures within the control group: $>99 \%$ for ISI 2 and
A

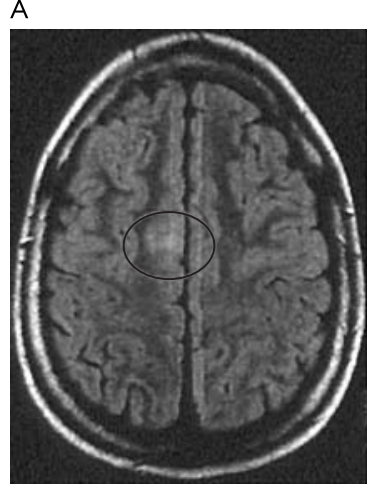

B

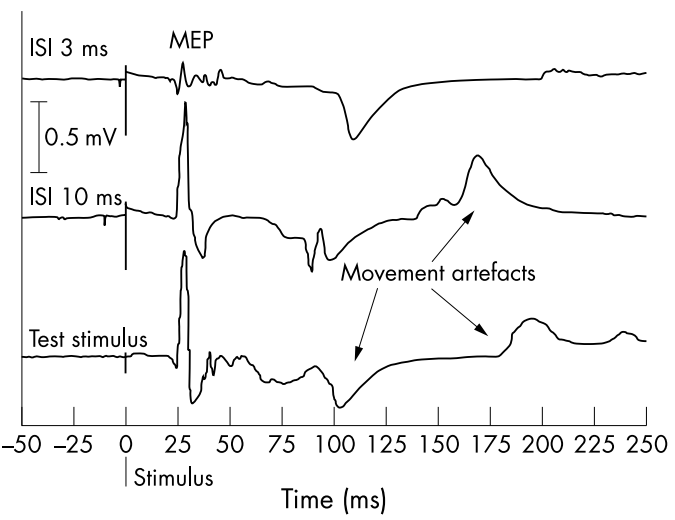

Figure 1 (A) Axial T2-weighted magnetic resonance imaging (MRI) scan (fluid attenuated inversion recovery (FLAIR)) of the patient's brain. T2 prolongation and blurring of grey-white junction represents focal cortical dysplasia of $1 \times 1 \mathrm{~cm}$ (see circle) located in the right superior frontal sulcus (extending to the right motor cortex, seen in further MRI slices). (B) Examples of motor evoked potentials (MEPs) from the right abductor digiti minimi muscle (ADM) during transcranial magnetic stimulation of the left hemisphere at different interstimulus intervals (ISI) and after unconditioned test stimulus. The patient showed stimulus triggered myoclonic jerks of both hands and forearms leading to movement artefacts in the ADM recordings.
CSP, $>95 \%$ for motor thresholds and ISI 15 , $>90 \%$ for ISI 10 , and $>85 \%$ for ISI 3 ).

After the TMS experiment, the patient was again free of daytime seizures until the last follow up visit six months later.

\section{Transcallosal seizure induction by paired pulse TMS}

In patients with epilepsy, all reported cases of seizure induction by TMS have occurred during ipsilateral stimulation and near to the epileptic focus. Therefore, it has been assumed that direct stimulation of the epileptogenic tissue was required to trigger a seizure. ${ }^{1}$ We used a focal coil placed over the left $\mathrm{Ml}$ hand area more than $5 \mathrm{~cm}$ away from the midline. Thus, it is unlikely that the right epileptogenic zone or mesial frontal cortex of the present patient was stimulated directly, and we assume that an indirect transcallosal activation of the epileptogenic zone provoked the aura. The latency of $65-75 \mathrm{~ms}$ of the myoclonic jerks after the MEP may reflect polysynaptic pathways in addition to a direct transcallosal connection of both $\mathrm{Ml}$. It is still not clear whether involvement of additional cortical areas such as the ipsilesional and contralesional sensory cortices or basal ganglia contributed to the seizure provocation. Despite the patient's statement that the jerks were not volitional, this cannot be completely ruled out. The preceding somatosensory aura, however, represented his typical seizure semiology. We hypothesise that transcallosal activation of the epileptic focus was promoted by the increased excitability of Ml, which was due to the underlying FCD. This, in turn, led to the aura and peri-ictal changes in Ml excitability facilitating TMS driven myoclonic jerking. It has been previously reported that FCD is intrinsically epileptogenic and promotes reflex seizures.

There is a possibility that ipsilateral pathways of movement activation could underlie our observations. In a child with extensive cortical dysplasia, TMS of the unaffected hemisphere evoked MEPs in both ADM muscles implying bilateral corticospinal connections from one cortex. ${ }^{4}$ Histological studies on severe brain damage in early development have revealed collateral sprouting into denervated areas of cortex or spinal cord. ${ }^{4}$ Ipsilateral activation under maximum muscle contraction has been observed in healthy volunteers and in patients with acute stroke. ${ }^{5}$ Our patient, however, presumably had congenital but circumscribed FCD, no motor deficits, and was investigated at rest. This and the fact that his habitual somatosensory aura occurred before the myoclonic jerks strongly argue against the activation of ipsilateral corticospinal tracts. Activation of a silent mirror focus in the left hemisphere with subsequent spread to the right is also unlikely because exclusively right sided ictal and interictal epileptiform discharges were recorded during the video-EEG monitoring.

\section{Changes in motor cortex excitability} Our patient's higher motor thresholds compared with controls are very likely due to his ion channel blocking anticonvulsant medication. ${ }^{6}$ 
The loss of intracortical inhibition and increased intracortical facilitation in the left hemisphere contralateral to the epileptogenic zone may reflect synaptic reorganisation of the ipsilesional and contralesional motor cortices. These distant functional cortical changes associated with malformations of cortical development have also been described previously. ${ }^{7}$ The prolongation of the cortical induced silent period seen in the present patient may be independent of the phenobarbital intake ${ }^{6}$ and confirms similar findings from previous studies as a remote effect of FCD on the motor cortex in untreated patients with cortical dysgenesis.

\section{Conclusion}

Unilateral epileptogenic FCD involving $\mathrm{Ml}$ can induce complex bilateral alteration of motor cortex excitability resulting in a net increase of excitability. In such cases, transcallosal seizure induction appears to be possible with paired pulse TMS using a focal coil away from the epileptic focus.

J Reis, F Rosenow, B Fritsch, S Knake, W H Oertel, H M Hamer

Interdisciplinary Epilepsy-Center, Department of Neurology, Philipps-University Marburg, Germany

Correspondence to: J Reis, Interdisciplinary EpilepsyCenter, Department of Neurology, Philipps-University Marburg, Rudolf-Bultmann-Str. 8, 35033 Marburg, Germany; reis@staff.uni-marburg.de

doi: 10.1136/jnnp.2004.042127

Competing interests: none declared

\section{Reference}

1 Classen J, Witte OW, Schlaug G, et al. Epileptic seizures triggered directly by focal transcranial magnetic stimulation. Electroencephalogr Clin Neurophysiol 1995;94:19-25.

2 Reis J, Tergau F, Hamer HM, et al. Topiramate selectively increases motor cortex excitability in human motor cortex. Epilepsia 2002;43: 1149-56.

3 Palmini A, Gambardella A, Andermann F, et al. Intrinsic epileptogenicity of human dysplastic cortex as suggested by corticography and surgical results. Ann Neurol 1995;37:476-87.

4 Maegaki Y, Yamamoto T, Takeshita K. Plasticity of central motor and sensory pathways in a case of unilateral extensive cortical dysplasia: investigation of magnetic resonance imaging transcranial magnetic stimulation, and shortlatency somatosensory evoked potentials. Neurology 1995;45:2255-61.

5 Caramia MD, Palmieri MG, Giacomini P, et al. Ipsilateral activation of the unaffected motor cortex in patients with hemiparetic stroke. Clin Neurophysiol 2000;111:1990-6.

6 Ziemann U, Lonnecker S, Steinhoff BJ, et al. Effects of antiepileptic drugs on motor cortex excitability in humans: a transcranial magnetic stimulation study. Ann Neurol 1996;40:367-78.

7 Cincotta M, Borgheresi A, Guidi L, et al. Remote effects of cortical dysgenesis on the primary motor cortex: evidence from the silent period following transcranial magnetic stimulation. Clin Neurophysiol 2000;1 11:1340-5

\section{Hashimoto's encephalopathy: steroid resistance and response to intravenous immunoglobulins}

Hashimoto's encephalopathy is a steroid responsive disorder characterised by high titres of anti-thyroid antibodies and manifesting as sub-acute onset of confusion, episodes of myoclonus, seizures, and strokelike episodes. Although excellent response to steroids is characteristic, other treatments such as plasmapheresis or administration of azathioprine or cyclophosphamide have been occasionally tried. We report a case of initially steroid responsive Hashimoto's encephalopathy which became steroid resistant and then responded well to intravenous immunoglobulins.

\section{Case report}

A 29 year old woman was admitted in 1987 with an episode of headache, confusion, agitation, and hallucination. She had a mild fever and was thought to have neck stiffness. A CT scan was normal as were the inflammatory markers. CSF examination showed 9240 red cells and 33 white cells (45\% polymorphs and 55\% lymphocytes). CSF protein, glucose, and microbiology were normal. A presumed diagnosis of meningoencephalitis was made and the patient was treated with acyclovir and antibiotics. The patient made a good recovery but was readmitted a week later with agitation and confusion with pain and weakness down the left side. No focal neurology was found on examination and the patient was thought to be suffering from an anxiety state.

During the next 14 years, the patient was admitted on several occasions with episodes of confusion and agitation: investigations including lumbar puncture, CT scans, EEG, thyroid function, porphyria screens, autoantibody screens (including antinuclear antibodies, ANCA, and those against extractable nuclear antigens), and metabolic and septic screens were found to be normal. She had been admitted to the psychiatry unit and was thought to be suffering from acute mania or a dissociative state, precipitated by stress and sleep deprivation. In 2001, she was referred to the neurology clinic for similar episodes, which were increasing in frequency.

On first review in the neurology clinic, clinical examination was unremarkable except for bilaterally symmetrical and brisk reflexes. MRI scan of the brain and EEG were repeated and found to be normal. Thyroid peroxidase antibody was raised at $250 \mathrm{IU} / \mathrm{ml}$ (normal range 0-60). Thyroid function tests were normal.

She remained well until April 2003 when a further episode of drowsiness and confusion occurred (thyroid peroxidase antibody $266 \mathrm{IU} / \mathrm{ml}$ ). This responded remarkably and within $24 \mathrm{~h}$ to a course of dexamethasone given intravenously at a dose of $16 \mathrm{mg}$ daily for 5 days. An EEG repeated during the episode showed diffuse slowing but no epileptiform abnormalities.

The patient was re-admitted in May 2004 with another episode of confusion and agitation. Interestingly, this episode occurred after a 5 day course of oral prednisolone $60 \mathrm{mg} /$ day for a chest infection. Apart from the acute confusional state there was no focal neurology. Repeat CT scan and CSF examination including oligoclonal bands were within normal limits. Repeat thyroid peroxidase antibody was elevated at $272 \mathrm{IU} / \mathrm{ml}$ (normal range $0-60$ ). Voltage gated potassium channel antibodies were negative. Other investigations including thyroid function remained normal. EEG showed diffuse bilateral slow wave activity. A 7 day course of intravenous dexamethasone $16 \mathrm{mg} /$ day was initiated. The confusion and agitation worsened and later she became very drowsy. Intravenous immunoglobulin ( $400 \mathrm{mg} / \mathrm{kg}$ daily) was given causing a dramatic improvement within $12 \mathrm{~h}$. Unfortunately, on the same day, the patient developed an increase in alanine transaminase and C-reactive protein levels. A possible reaction to intravenous immunoglobulin was considered and the infusion was discontinued. Later, the increased levels were confirmed to be secondary to septicaemia from a Staphylococcus aureus infected cannula site. The inflammatory markers normalised after a course of antibiotics. During this time the patient remained drowsy, confused, and occasionally agitated. A 5 day course of intravenous immunoglobulin was reinstated. Again, there was a dramatic improvement within $24 \mathrm{~h}$ and she was discharged home at the end of the course having completely recovered.

\section{Discussion}

Hashimoto's encephalopathy (HE) is a steroid responsive disorder characterised by high titres of anti-thyroid antibodies. The original description of this condition was in an established case of Hashimoto's thyroiditis where the patient developed focal neurological deficits and coma. ${ }^{1}$ Clinical presentation includes episodic confusion, myoclonus, seizures, and stroke-like episodes. ${ }^{2}$ Females are more affected than males (3.6:1), with a mean age of onset of 41 years. The hallmark of $\mathrm{HE}$ is its response to steroids, most cases improving within a few hours to days. ${ }^{3}$ The titres of anti-thyroid antibodies maybe independent of the severity of the clinical presentation. ${ }^{4}$ Fewer than 100 cases of HE have been reported in the literature. Goitre and hypothyroidism can be associated with the disorder, but the majority of patients are euthyroid. Although steroid responsiveness is the rule, additional immunosuppressive therapy in the form of azathioprine and cyclophosphamide has been tried in a minority of patients. ${ }^{23}$ One case report of HE responding to plasmapheresis is documented. ${ }^{5}$ There was no benefit in the two documented cases where intravenous immunoglobulin was administered. $^{56}$

Several pathophysiological hypotheses have been suggested for HE. The initial report of HE suggested a vascular aetiology followed by localised cerebral oedema as a possible mechanism. ${ }^{1}$ Some authors suggest that the CSF thyroid autoantibodies may react with a putative CNS antigen and form immune complexes. $^{24}$ The immunopathological basis of this syndrome has been compared to a relapsing form of acute disseminated encephalomyelitis. ${ }^{7}$ Although reversible MRI findings have been described in $\mathrm{HE}^{8}$ neuroimaging (except for isolated patchy uptake by isotope scans) is usually normal in most cases. ${ }^{3}$ Cerebral angiography has been found to be normal in several cases of HE, unlike in many other cerebral vasculitides. ${ }^{1-3} 5$

Thyroid autoantibodies can co-exist with several other forms of autoimmune encephalomenigitis, but the normal MRI scan, the initial dramatic response to steroids, and negative autoantibodies for most other common vasculitides, tends to favour the diagnosis of $\mathrm{HE}$ in our case. Steroid responsive encephalopathy associated with Hashimoto's thyroiditis is an alternately proposed name for this condition, ${ }^{9}$ but the vast majority of cases have normal thyroid function, leaving "Hashimoto's encephalopathy" a universally accepted term. A recent literature review of 85 patients with encephalopathy and antithyroid antibodies suggests that the combination of encephalopathy, high serum anti-thyroid antibody concentrations, and 
responsiveness to glucocorticoid therapy seems unlikely to be due to chance. ${ }^{10}$

The initial meningo-encephalitic type presentation of our patient in 1987 was probably the first manifestation of $\mathrm{HE}$ in view of clinical findings and laboratory data (Mild CSF pleocytosis is not unusual in HE. ${ }^{3}$ ) There was a delay of 14 years before the diagnosis was first established, in spite of several hospital admissions. The initial relapses after diagnosis responded well to steroids, confirming the diagnosis of HE. Whether the current episode was precipitated by the sudden withdrawal of oral steroids or the chest infection itself, for which they were prescribed, is unclear.

Our patient illustrates the possibility of steroid resistance in an established case of $\mathrm{HE}$ and the need to consider further immunomodulatory therapy. Intravenous immunoglobulins are a safe, convenient, and effective treatment in such circumstances.

S Jacob, Y A Rajabally Department of Neurology, Leicester Royal Infirmary, University Hospitals of Leicester, Leicester LE1 5WW

Correspondence to: $\operatorname{Dr}$ Y A Rajabally, Department of Neurology, University Hospitals of Leicester, Royal Infirmary, Leicester LE1 5WW, UK; yusuf.rajabally@ uhl-tr.nhs.uk

doi: 10.1136/jnnp.2004.049395

Competing interests: none declared

\section{References}

1 Lord Brain, Jellinek EH, Ball K. Hashimoto's disease and encephalopathy. Lancet 1966;2:512-4.

2 Shaw PJ, Walls TJ, Newman PK, et al. Hashimoto's encephalopathy: a steroidresponsive disorder associated with high antithyroid antibody titre - report of 5 cases. Neurology 1991;41:228-33.

3 Peschen-Rosin R, Schabet M, Dichgans J. Manifestation of Hashimoto's encephalopathy years before onset of thyroid disease. Eur Neurol 1999:41:79-84

4 Ferraci F, Moretto G, Candeago RM, et al. Antithyroid antibodies in the CSF. Their role in the pathogenesis of Hashimoto's encephalopathy. Neurology 2003;60:712-4.

5 Boers PM, Colebatch JG. Hashimoto's encephalopathy responding to plasmapheresis. J Neurol Neurosurg Psychiatry 2002;73:601.

6 Henchey R, Cibula J, Helveston W, et al. Electroencephalographic findings in Hashimoto's encephalopathy. Neurology 1995;45:977-81.

7 Chaudhuri A, Behan PO. The clinical spectrum, diagnosis, pathogenesis and treatment of Hashimoto's encephalopathy (recurrent acute disseminated encephalomyelitis). Curr Med Chem 2003; 10(19): 1945-53

8 Bohnen NI, Parnell KJ, Harper CM. Reversible MRI findings in a patient with Hashimoto's encephalopathy. Neurology 1997;49:246-7.

9 Mahmud FH, Renaud DL, Reed AM, et al. Steroidresistant encephalopathy associated with Hashimoto's thyroiditis in an adolescent with chronic hallucinations and depression: case report and review. Pediatrics 2003; 112(3 Pt 1):686-90.

10 Chong JY, Rowland LP, Utiger RD. Hashimoto's encephalopathy. Syndrome or myth? Arch Neurol 2003:60:164-71

\section{Spontaneous lobar haemorrhage in CADASIL}

CADASIL is an autosomal dominant form of arteriopathy, primarily affecting cerebral vessels, and predominantly caused by point mutations in the Notch 3 gene on the short arm of chromosome 19. ${ }^{1}$ Affected individuals develop subcortical strokes and cognitive deficits in their 50s and $60 \mathrm{s.}^{2}$ Brain magnetic resonance imaging (MRI) shows large areas of leukoencephalopathy and multiple subcortical lacunar infarcts. Small arteries and capillaries are characterised histologically by a non-atherosclerotic, non-amyloid angiopathy with accumulation of granular osmiophilic material (GOM) within the smooth muscle cell basement membranes and extracellular matrix. ${ }^{3}$ While CADASIL is considered a primarily ischaemic form of vascular dementia, microhaemorrhages have recently been reported in $31 \%$ of symptomatic Notch 3 mutation carriers, suggesting that structural fragility of the arterial walls may lead to leaking of haem products. ${ }^{4}$ Lobar haemorrhage in the absence of other risk factors for haemorrhage has previously been reported in one patient with CADASIL. ${ }^{5}$ Here we report a second case.

\section{Case report}

A 56 year old man who had been diagnosed with multiple sclerosis six years earlier was admitted to the hospital with an acute change in mental state. He had collapsed at home and was unresponsive when rescue arrived. In the emergency room he had a depressed level of consciousness and difficulty following commands, with paucity of speech, dysarthria, and hypophonia. There was no evidence of head trauma. His blood pressure was $100 / 63 \mathrm{~mm} \mathrm{Hg}$ and his temperature was $36.1{ }^{\circ} \mathrm{C}$

Past medical history included chronic obstructive pulmonary disease, prostate resection for prostate cancer, and a history of nicotine and alcohol dependence. He had no history of hypertension, diabetes mellitus, or coagulopathy. His drug treatment included ipratropium, ranitidine, methyprednisolone, and albuterol. His mother, now deceased, had been diagnosed as having multiple sclerosis and had migraines with auras, stroke-like symptoms, and dementia. He had eight siblings, three with headaches and one with recent transient ischaemic events.

Computed tomography (CT) of the head in the emergency department showed an area of high attenuation in the right frontal lobe consistent with an acute intraparenchymal haemorrhage (fig lA). There was no evidence of trauma on head CT. Gradient echo MRI sequences of the brain done on hospital day 2 showed a $2 \times 2.5 \mathrm{~cm}$ area of haemorrhage in
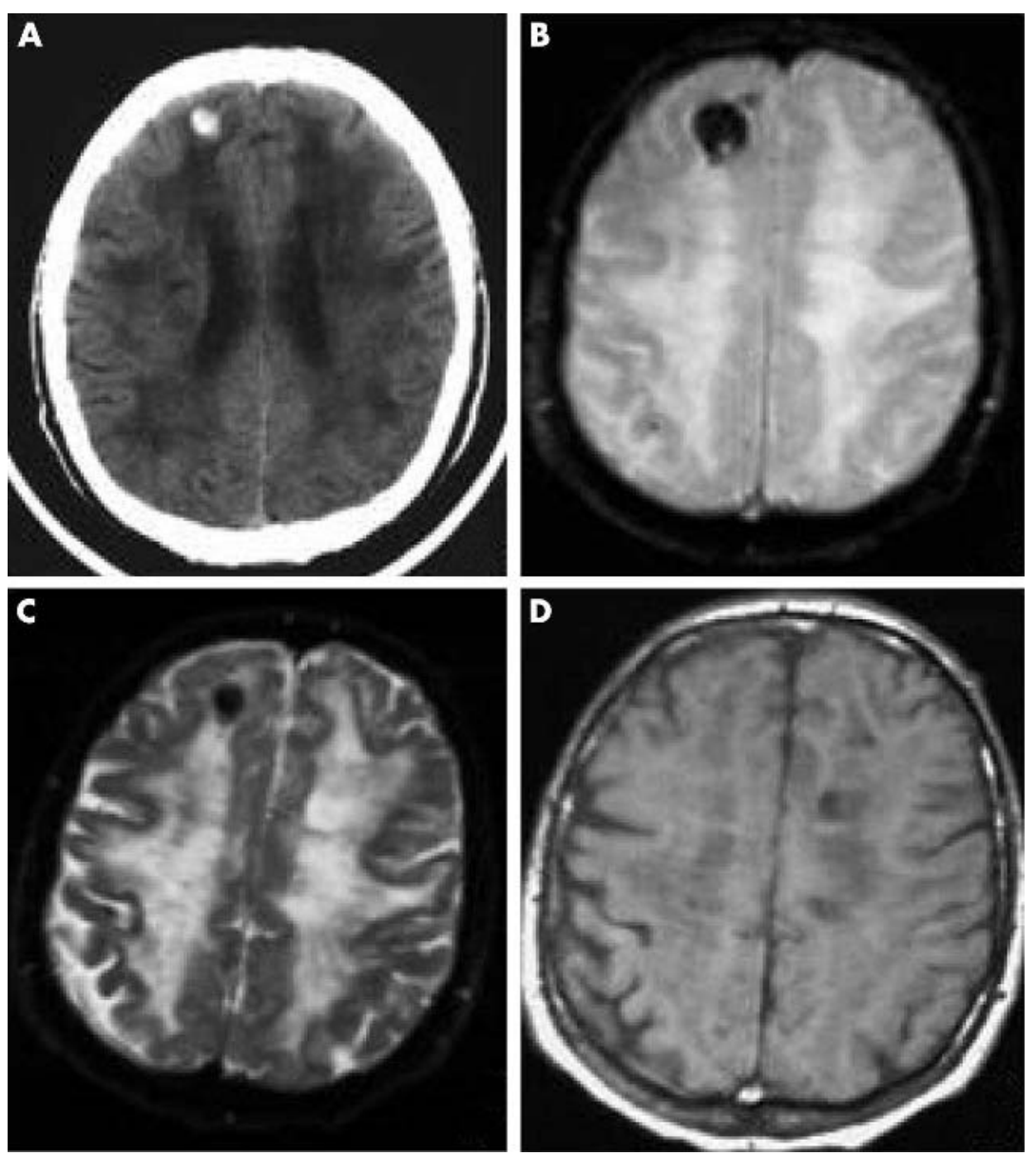

Figure 1 (A) Non-contrast computed tomography of the head done in the emergency room showing an area of high attenuation in the right frontal lobe consistent with acute haemorrhage. The other panels show non-contrast magnetic resonance imaging done on hospital day 2 : (B) Gradient echo sequence demonstrating a $2 \times 2.5 \mathrm{~cm}$ area of haemorrhage in right frontal lobe, a microhaemorrhage in the right parietal region, and extensive white matter disease. (C, D) The area of haemorrhage is hypointense on T2 and isointense on $\mathrm{T} 1$ weighted imaging, consistent with an acute haemorrhage. 
the superior-anterior aspect of the right frontal lobe white matter as well as a microhaemorrhage in the right parietal region (fig $1 \mathrm{~B}$ ). The area of haemorrhage was hypointense on T2 (fig IC) and isointense on $\mathrm{Tl}$ weighted sequences (fig ID), consistent with acute haemorrhage. There was no MRI evidence of a cavernous haemangioma, arteriovenous malformation, or tumour. Magnetic resonance angiography was not done.

A brain biopsy of the right frontal lobe done on the seventh hospital day showed degeneration of small and medium sized arteries. Vessel walls were thick and hyalinised in the grey matter, white matter, and meninges. PAS staining was positive and the muscular coat of the large vessels revealed degenerative changes. Electron microscopy showed the granular osmiophilic material characteristic of CADASIL. Notch 3 gene testing revealed a R133C mutation in exon 4, consistent with the diagnosis of CADASIL. The patient remained normotensive throughout his hospital stay. On the fifth hospital day he developed aspiration pneumonia requiring mechanical ventilation. He died eight days later as a result of this pneumonia.

\section{Comment}

This is the second report of spontaneous cerebral haemorrhage in a patient with CADASIL. In 1977, Sourander and Walinder reported a 29 year old man with hereditary multi-infarct dementia on anticoagulants, with a large haemorrhage in the right hemisphere. ${ }^{5}$ This family was thought to be one of the first with CADASIL; however, recent testing for Notch 3 mutations in the family has not confirmed that diagnosis. ${ }^{6}$ In 1992, Baudrimont et al reported a case of massive left cerebral haematoma involving the caudate nucleus, internal capsule, and thalamus in a 40 year old normotensive woman who was a member of a large CADASIL family. She had no known history of other risk factors for haemorrhage. ${ }^{7}$

The index patient in this report had no evidence of coagulopathy and no history of previous hypertension, cerebral haemorrhage, or anticoagulant therapy. The patient could have experienced a haemorrhagic contusion related to a closed head injury during his unwitnessed fall before admission, but there was no evidence of trauma on physical examination or on head CT. On MRI there was no evidence of a cavernous haemangioma, arteriovenous malformation, or neoplasm. Necropsy was not carried out.

Ultrastructural analysis of small arteries in human postmortem brain and skin in patients with CADASIL shows breakdown of the arterial wall cytoarchitecture, which may help explain the propensity for microhaemorrhages. ${ }^{8}$ The first notch 3 transgenic mouse shows early widening of the subendothelial and intra-smooth-muscle spaces in the vascular smooth muscle cells, denoting weakening of the arterial wall and increasing susceptibility to micro- and macrohaemorrhages. ${ }^{9}$

This case report supports the growing evidence for both ischaemia and haemorrhage in a variety of small artery diseases including amyloid angiopathy and CADASIL. ${ }^{10}{ }^{11}$ Clinicians may need to consider the possibility of haemorrhage when evaluating new events and deciding on treatment for stroke prevention in patients with CADASIL.
Acknowledgements

Supported by grants P20 RR015578 and K08MH001487.

A V Maclean

Brown Medical School, Providence, Rhode Island, USA

R Woods, L M Alderson, S P Salloway Department of Clinical Neurosciences Brown Medical School

S Correia

Department of Psychiatry and Human Behavior Brown Medical School

S Cortez, E G Stopa

Department of Pathology (Neuropathology Division) Brown Medical School

Correspondence to: Professor Stephen Salloway, Department of Neurology, Butler Hospital, 345 Blackstone Blvd, Providence, RI 02906, USA Stephen_Salloway@brown.edu

doi: 10.1136/jnnp.2004.042564

Competing interests: none declared

\section{References}

1 Joutel A, Corpechot C, Ducros A, et al. Notch3 mutations in CADASIL, a hereditary adult-onset condition causing stroke and dementia. Nature 1996;383:707-10.

2 Dichgans M, Mayer M, Uttner I, et al. The phenotypic spectrum of CADASIL: clinical findings in 102 cases. Annals of Neurology 1998;44:731-9.

3 Ruchoux MM, Maurage CA. CADASIL: cerebral autosomal dominant arteriopathy with subcortical infarcts and leukoencephalopathy. J Neuropathol Exp Neurol, 1997; 56:947-64.

4 Lesnik Oberstein SA, van den Boom R, van Buchem MA, et al. Cerebral microbleeds in CADASIL. Neurology 2001;57:1066-70.

5 Sourander P, Walinder J. Hereditary multi-infarct dementia: morphological and clinical studies of a new disease. Acta Neuropathol (Berlin) 1977:39:247-54.

6 Kalimo H, Ruchoux MM, Viitanen $M$, et al. CADASIL: a common form of hereditary arteriopathy causing brain infarcts and dementia. Brain Pathol 2002; 12:371-84.

7 Baudrimont M, Dubas F, Joutel A, et al. Autosomal dominant leukoencephalopathy and subcortical ischemic stroke: a clinicopathological study. Stroke 1993;24:122-5.

8 Rouchoux MM, Maurage CA. Endothelial changes in muscle and skin biopsies in patients with CADASIL. Neuropathol Appl Neurobiol 1998;24:60-5.

9 Ruchoux MM, Domenga $\mathrm{V}$, Brulin $\mathrm{P}$, et al. Transgenic mice expressing mutant notch 3 develop vascular alterations characteristic of cerebral autosomal dominant angiopathy with subcortical infarcts and leukoencephalopathy. Am J Pathol 2003;162:329-42.

10 Greenberg SM. Cerebral amyloid angiopathy and vessel dysfunction. Cerebrovasc Dis 2002; 13(suppl 2):42-7.

11 Dichgans M, Holtmannspotter M, Herzog J, et al. Cerebral microbleeds in CADASIL: a gradientecho magnetic resonance imaging and autopsy study. Stroke 2003;34:376-7

\section{Urinary retention caused by a small cortical infarction}

The cortical representation of micturition is speculated to reside in the medial frontal lobes. ${ }^{12}$ Lesion pathology, however, varies from acute stroke to a neoplasm, and there is not necessarily a small, distinct lesion. ${ }^{2}$ We report a case of urinary retention in which the main presenting symptom is thought to have been caused by a small cortical infarction.

\section{Case report}

One morning, a 66 year old, right handed man had difficulty urinating. He had no history of voiding difficulty, diabetes mellitus, injury to the lower urinary tract, or neurological disease. Digital rectal examination and ultrasonography of the prostate detected no enlargement. Urinalysis showed no haematuria or pyuria. He was not taking any medications that cause voiding dysfunction. There was no urinary incontinence, but he had difficulty in voiding even though he felt the bladder was full. At that time, he also had difficulty in lifting his left arm and leg and so was brought to our hospital. Neurological examination in the emergency room found no weakness, and he was sent home. Later, he experienced urinary retention and visited the emergency room again. His post void residual urine volume was $350 \mathrm{ml}$, and an urinary catheter was inserted. At that time the patient was alert, and his cranial nerves were intact. Limb muscle strength was normal. Sensory examination was unremarkable. Tendon reflexes were normal in all four limbs. Tandem gait and standing on one foot were difficult. He had normal bladder sensation but difficulty in urinating. Drip infusion pyelography revealed no abnormality in the upper urinary tract or the form of the bladder. Filling cystometry showed stable detrusor with normal bladder sensation, whereas acontractile detrusor was noted in the voiding phase. He could void only with strain, having a peak flow rate of $5.0 \mathrm{ml} / \mathrm{s}$ and a voided volume of $135 \mathrm{ml}$. Diffusion weighted MRI, performed on the day of onset, showed a small, distinct, high intensity signal, and $\mathrm{Tl}$ weighted imaging showed a low signal in the right caudal part of the anterior cingulate gyrus, indicative of an infarct in the acute stage (fig 1A and C). No definitive infarct was observed elsewhere. MR angiography showed no occlusion or stenosis of the intracranial vessels. An electrocardiogram was normal. Transthoracic echocardiograms showed no abnormal findings. The urinary catheter was withdrawn 3 days after admission, and he had no subsequent difficulty with urination. His gait returned to normal about the same time.

\section{Discussion}

In the acute stage of a cerebral vascular accident, the presenting symptom often is urinary retention due to detrusor areflexia, ${ }^{3}$ but patients who have this problem usually have a major stroke with severe neurological deficits.

To the best of our knowledge, this is the first report in the English literature of urinary retention, although temporary, caused by a small cortical infarct as shown by diffusion weighted MRI.

Various cortical areas are activated during voiding because a network of brain regions is necessary for voiding modulation. ${ }^{4}$ The locations of the primary cerebrum cortical areas for voiding and storage are speculated to be separate, the former being at the para-central lobule. ${ }^{2}$ A PET study found normal micturition to be associated with activation of the middle frontal gyrus, superior frontal gyrus, superior precentral gyrus, thalamus, and the caudal part of the anterior cingulate gyrus in the left hemisphere. ${ }^{5}$ Another recent PET study showed that increased brain activity related to increasing bladder volume was located in the bilaterally mid-cingulate cortex, while that related to decreased urge to 

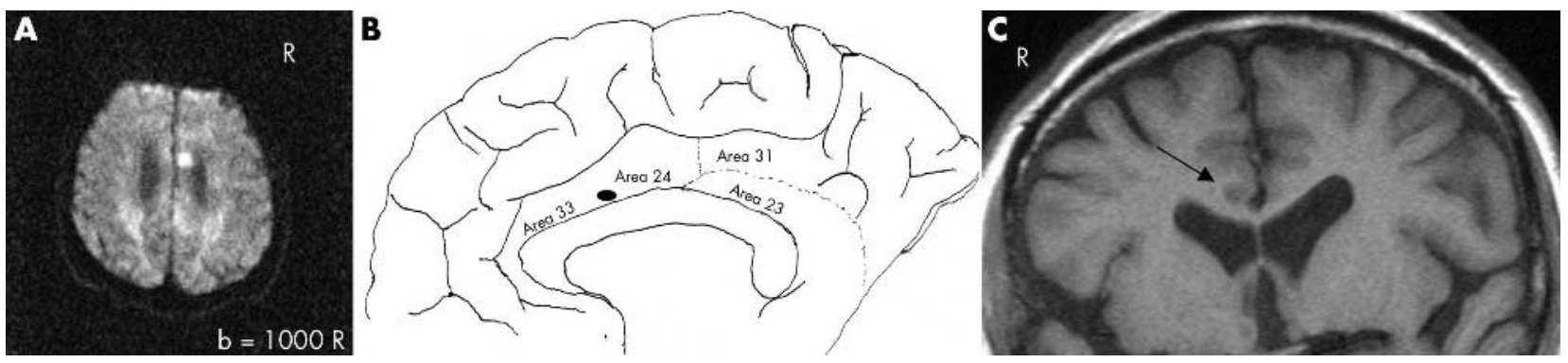

Figure 1 Diffusion weighted MRI. (A) The axial section shows a small high signal in the right caudal part of the anterior cingulate gyrus. (B) The closed ellipse denotes the lesion in the cortical map (map modified from Brodmann, 1909). (C) In the T1 weighted MRI procedure, the coronal section shows a low intensity signal in the right caudal part of anterior cingulate gyrus (arrow). (Imaging condition: a negative tilt of $-20^{\circ}$ to the orbitomeatal line.)

void was bilaterally in a different portion of the mid-cingulate gyrus. ${ }^{4}$

Although the infarct in our patient was located in the caudal part of the anterior cingulate gyrus, it was on the right side, nearby the region activated in the PET study. SPECT showed increased blood flow in the right medial frontal area, indicative that urinary retention was due to "decreased urge to void", and decreased flow in the right medial parietal lobe, which might explain the gait disturbance, in light of the essentially normal sensory examination. Unfortunately, a PET scan was not available in our hospital (Kameda Medical Center). Because there has been no report of an isolated lesion of the cingulate gyrus causing hemiparesis, these brain imaging studies indicate that the left hemiparesis, which disappeared within a half day of onset, could have been due to a transient ischaemic attack.

Urinary symptoms disappeared 3 days after admission, probably because the cortical neuron network compensated by providing a functional alternative to the lesion damaged by the infarct. This is similar to the condition of urinary incontinence after cerebral infarction, as is well documented. The laterality of the lesion in this patient differs from that in a previous PET study ${ }^{4}$ which showed bilateral activation in the cingulate gyrus. Because this report cites only a single case, its applicability is limited. Additional lesion studies of patients with micturition disturbance due to small cortical infarcts should help to identify the anatomical cerebral structures involved in voiding.

K Funakoshi, T Fukutake, H Nishino, S Sato Department of Neurology, Kameda Medical Center, Chiba, Japan

T Yamanishi

Department of Urology, Dokkyo University School of Medicine, Tochigi, Japan

Correspondence to: Dr Funakoshi, Department of Neurology, Dokkyo University School of Medicine, Kitakobayashi 880, Mibu, Shimotsuga, Tochigi 3210293, Japan; kei-f@dokkyomed.ac.jp

doi: 10.1136/jnnp.2004.050542

Competing interests: none declared

\section{References}

1 Sakakibara R, Hattori T, Yasuda K, et al. Micturitional disturbance after acute stroke: analysis of the lesion site by CT and MRI. J Neurol Sci 1996; 137:47-56.

2 Kuroiwa Y. Neurological bladder dysfunction due to frontal lobe lesions. Neurol Med (Tokyo) 1992;36:561-7.
3 Bradley WE. Cystometry and sphincter electromyography. Mayo Clin Proc 1976;51:329-35.

4 Athwal BS, Berkley KJ, Hussain I, et al. Brain responses to changes in bladder volume and urge to void in healthy men. Brain 2001;124:369-77.

5 Sam N, Claus S, Jorgen KI, et al. Cerebral activation during micturition in normal men. Brain 2000;123:781-9.

\section{BOOK REVIEW}

\section{Neuropsychiatry and behavioural neurology explained}

Edited by A J Mitchell. Published by Saunders, London, 2004, £69.00 (hardback), pp 500. ISBN 0-7020-2688-3

This is an ambitious project for a single author; the whole of neuropsychiatry explained using an up to date, evidence based review of the literature, and in a format that is designed to be attractive to read. There are numerous figures, boxes, lists with bullet points, and "clinical pointers" to break up the text.

Although aimed particularly at liaison and old age psychiatrists, this book will have wide appeal and be of interest to neurologists. They will be able to quickly access clinically relevant discussion of the neuropsychiatric sequelae of common neurological disorders. The core sections of the book, on dementia and delirium, neuropsychiatric treatments, and the psychiatric complications of neurological diseases, are excellent. The discussion is practical and to the point. The reader is not stifled with references strewn in the text. They must therefore have confidence in the assertions of the author; I am confident that we are being offered accurate information. But at times the style feels a little pedantic; for example, those of us who dared to believe that alcohol might cause depression are put firmly in our place. Another quibble I have is the value of some of the lists/classifications which were of uncertain provenance. We are, for example, given lists suggesting difference aetiologies for chorea versus athetosis, but some would be sceptical of the value in splitting choreoathetosis. Many classifications are based on neuroanatomical models of neuropsychiatry that need to be treated with caution.

The book strays into biological psychiatry, and a later section is devoted to understanding how neurological disorders result in neuropsychiatric symptoms, but this does cause a problem because some of the discussion of the neuropsychiatric sequelae of a particular disorder may not be found in the index chapter on that disorder, but in this later section. For example, the only discussion of suicide following head injury in the chapter on head injury is a single misleading sentence indicating that suicide accounts for $10 \%$ of head injury deaths. Yet, easily missed, 300 pages later, in the chapter on the neurological origins of suicide, is a more complete account of the relationship.

Overall, however, this book is a significant achievement. A large amount of material has been made readily accessible. There are no lacunae and the length of discussion of each disorder is proportionate to its importance. The book is to be trusted and recommended One interesting innovation is a list of support groups and useful websites in the appendix. Neurologists and psychiatrists and their trainees have good reason to buy this book.

S Fleminger

\section{CORRECTIONS}

doi: 10.1136/jnnp.2004.047118corr l

Wood J M, Worringham C, Kerr G, et al Quantitative assessment of driving performance in Parkinson's disease ( $J$ Neurol Neurosurg Psychiatry 2005; 76:176-80). SD instead of SE was inadvertently inserted during the publication process in tables 2,3 , and 4 . The $\mathrm{p}$ values are unaffected.

doi: 10.1136/jnnp.2003.029389corr l

Barber PA, Demchuk AM, Hill MD, et al. The Probability of middle cerebral artery MRA flow signal abnormality with quantified CT ischaemic change: targets for future therapeutic studies ( $J$ Neurol Neurosurg Psychiatry 2004;75:1426-30). The following errors appeared in this article:

(1) The median CT ASPECTS and DWI ASPECTS quoted in the article were both 8. These are incorrect and should be CT ASPECTS 9 and DWI ASPECTS 8 ;

(2) Sixty-six per cent (95\% CI $0.56-0.75)$ of the patients had CT ischaemic change, while $81 \%$ (95\% CI $0.72-0.88)$ of the DWI scans identified areas of hyperintense signal (not $67 \%$ and $79 \%$ quoted in the article);

(3) In figure 2 the numbers in parentheses on the $\mathrm{x}$ axis were incorrect. The correct numbers for each ASPECTS value are 10 (34), $9(21), 8(12), 7(11), 6(12)$, and 5 (10). 